\title{
Elevated plasma Sirtuin2 level predicts heart failure after acute myocardial infarction
}

\author{
Meili Zheng ${ }^{1,2 \#}$, Xiangpeng Du ${ }^{3 \#}$, Lei Zhao ${ }^{1,2 \#}$, Hao Sun $^{1}$, Mulei Chen ${ }^{1}$, Xinchun Yang ${ }^{1,2}$ \\ ${ }^{1}$ Heart Center, Beijing Chao-Yang Hospital, Capital Medical University, Beijing, China; ${ }^{2}$ Beijing Key Laboratory of Hypertension Research, \\ Beijing Chao-Yang Hospital, Capital Medical University, Beijing, China; ${ }^{3}$ Department of Cardiology, Weihaiwei People's Hospital, Weihai, China \\ Contributions: (I) Conception and design: X Yang, M Chen, H Sun; (II) Administrative support: X Yang; (III) Provision of study materials or patients: \\ X Yang, M Chen; (IV) Collection and assembly of data: M Zheng, X Du, L Zhao; (V) Data analysis and interpretation: M Zheng, X Du; (VI) \\ Manuscript writing: All authors; (VII) Final approval of manuscript: All authors. \\ \#These authors contributed equally to this work. \\ Correspondence to: Prof. Xinchun Yang, Prof. Mulei Chen, Dr. Hao Sun. Heart Center, Beijing Chao-Yang Hospital, Capital Medical University, 8\# \\ Gong-Ti South Road, Beijing, China. Email: yangxc99@gmail.com; cml68@sina.cn; sunhaosub@163.com.
}

Background: There is currently no evidence regarding the role of plasma Sirtuin2 (SIRT2) level in acute myocardial infarction (AMI) yet. This study assessed the role of plasma SIRT2 in AMI, and investigated the association of plasma SIRT2 level with major adverse cardiovascular events (MACE) and heart failure after AMI.

Methods: This is a prospective observational study. A total of 129 AMI patients (mean age: $62.2 \pm 12.7$ years old, male/female: 96/33) were included. Cox proportional hazards regression models were used to estimate the association of different SIRT2 levels with MACE and heart failure after AMI.

Results: According to the 75th percentile value of plasma SIRT2 level, we divided all the AMI patients into two groups: high-level group (plasma SIRT2 level $\geq 109.0 \mathrm{pg} / \mathrm{mL}$ ) and low-level group (plasma SIRT2 level $<109.0 \mathrm{pg} / \mathrm{mL}$ ). Compared with the low-level group, the high-level group had higher percentage of Killip class $\geq 3(\mathrm{P}<0.001)$, left ventricular ejection fraction (LVEF) $<50 \%(\mathrm{P}=0.007)$ or even $<40 \%(\mathrm{P}=0.012)$, use of breathing machine $(\mathrm{P}=0.003)$, and higher plasma brain natriuretic peptide $(\mathrm{BNP})$ level $(\mathrm{P}=0.006)$. Multivariate Cox regression analysis showed that there were higher risks of MACE [hazard ratio (HR) 11.20, 95\% confidence interval $(\mathrm{CI}): 3.18-39.52, \mathrm{P}<0.001)]$ and heart failure (HR 27.10, 95\% CI: 4.65-157.83, $\mathrm{P}<0.001)$ in the high-level group.

Conclusions: The present study suggested that plasma SIRT2 level is a promising biomarker to predict heart failure and MACE after AMI.

Keywords: Sirtuin2 (SIRT2); acute myocardial infarction (AMI); ST-segment elevation myocardial infarction (STEMI); non-ST-segment elevation myocardial infarction (NSTEMI)

Submitted Jun 17, 2020. Accepted for publication Oct 25, 2020.

doi: $10.21037 /$ jtd-20-2234

View this article at: http://dx.doi.org/10.21037/jtd-20-2234

\section{Introduction}

Sirtuin (SIRT) is a family of $\mathrm{NAD}^{+}$-dependent histone deacetylases, regulating metabolism and aging-related diseases (e.g., diabetes, cancer, neurodegenerative and cardiovascular diseases) (1-5). Recently, SIRT2, as a member of sirtuin family, was reported to play a significant role in cardiovascular disease. It has been previously shown that over expression of cardiac-specific SIRT2, promoting AMP-activated protein kinase (AMPK) activation, can protect heart against Ang II-induced cardiac hypertrophy and fibrosis (6), while SIRT2 can repress nuclear factor of 
activated T-cells (NFAT) to maintain cardiac homeostasis and ameliorate cardiac dysfunction (7). In addition, SIRT2 gene is down-regulated in the cardiac tissue in cardiosurgical patients undergoing remote ischemic preconditioning (8), and functional genetic variants in acute myocardial infarction(AMI) patients were observed as well (9).

It is noteworthy that, SIRT2 is expressed in various metabolically relevant tissues (e.g., the heart, brain, and adipose tissue) (10), and is also detected in circulation (11). SIRT2 plays a pivotal role in various physiological processes in maintaining metabolic homeostasis, including inflammation, oxidative stress, and mitochondrial function, as well as adipocyte differentiation, fatty acid oxidation, and insulin sensitivity. SIRT2 may enhance acetylation and activation of NF-kB p65 $(12,13)$ and regulate expression of CXCL2 and CCL2 (14) to suppress inflammatory process, while regulate acetylation of G6PD to modulate NADPH homeostasis and cell survival during oxidative stress (15). Dysregulated SIRT2 activity has been found to be associated with inflammatory and metabolic disorders $(10,16)$. Moreover, AMI, leading to the highest mortality among cardiovascular diseases, is involved in both metabolic dysfunction and inflammatory responses (17-19). The cause of AMI patients' death is either heart failure or a malignant arrhythmia, especially heart failure, which is closely associated with inflammatory responses and contributes to long-term mortality after AMI (20,21).

However, no study has concentrated on the role of circulating SIRT2 in AMI yet. In the present study, we investigated the relationship between plasma SIRT2 level and AMI, and evaluated the association of plasma SIRT2 level with major adverse cardiovascular events (MACE) and heart failure after AMI. Our results clarified the role of plasma SIRT2 level in AMI prognosis. We present the following article in accordance with the STROBE reporting checklist (available at http://dx.doi. org/10.21037/jtd-20-2234).

\section{Methods}

\section{Study subjects}

This is a prospective observational study. Study subjects were consecutively recruited from Beijing Chao-yang Hospital (Beijing, China) between October 2018 to March 2019. A total of 129 AMI patients [including 74 ST-segment elevation myocardial infarction (STEMI) and 55 non-ST- segment elevation myocardial infarction (NSTEMI)] with heart attack within 12 hours were enrolled in the present study. All patients successfully underwent revascularization in emergency before hospitalization. The diagnosis of AMI was carried out at the time of admission on the basis of criteria, including clinical symptoms, typical changes in electrocardiogram (ECG), elevated cardiac biomarkers (cardiac troponin-I and creatine kinase $\mathrm{MB}$ ). The exclusion criteria were as follows: neoplasm, severe organ failure, or other infectious or inflammatory conditions. Written informed consent was obtained from all the participants. This study was conducted in accordance with the Declaration of Helsinki (as revised in 2013) (22), and the research protocol was approved by the Ethics Committee of Beijing Chao-Yang Hospital (No. 2018-2-7-3) and informed consent was taken from all the patients.

\section{Clinical conditions}

For all the AMI patients data related to cardiac arrest, utilization of intra-aortic balloon pump (IABP), and breathing machine, and death during hospitalization were recorded, and all the patients were followed-up for 12 months. The MACE included cardiac death, readmission for revascularization and heart failure. Heart failure involved death due to heart failure during hospitalization, and readmission because of heart failure after discharge.

\section{Laboratory measurements}

Baseline laboratory measurements were obtained within the first 12 hours of admission. Plasma of SIRT2 levels were assayed using a commercially available enzyme-linked immunosorbent assay (ELISA) kit (R\&D Systems Inc., Minneapolis, MN, USA) according to the manufacturer's instructions. The coefficient of variation for the assay was $<5 \%$. Fasting venous blood samples were collected to measure the levels of glucose, homocysteine, creatinine, and lipids (including the levels of total cholesterol, high-density lipoprotein cholesterol, and triglycerides).

\section{Statistical analysis}

Continuous variables were expressed as mean \pm standard deviation (SD) and quartiles, while categorical variables were expressed as percentages and numbers. Comparisons between groups were performed using chi-square test for categorical variables, and two-sample $t$-test for comparing 
continuous variables in normally distributed status, as well as Kruskal-Wallis test for comparing continuous variables in non-normally distributed status. Pearson's and Spearman's correlation coefficients were used for comparing parametric and nonparametric variables, respectively. Cox proportional hazards analysis was carried out to determine the independent predictors of MACE. All the analyses were performed using SPSS 24.0 software (IBM, Armonk, NY, USA), and a 2 -tailed $\mathrm{P}<0.05$ was considered statistically significant.

\section{Results}

\section{Study subjects' clinical characteristics}

A total of 129 AMI patients (mean age: $62.2 \pm 12.7$ years old, male/female: 96/33) were enrolled in the present study, including 74 STEMI and 55 NSTEMI. The median follow-up period was 8 months, and during the follow-up, $22(17.1 \%)$ and $16(12.4 \%)$ AMI patients experienced MACE and heart failure respectively. The median value $\left(25^{\text {th }}, 75^{\text {th }}\right.$ percentiles) of plasma SIRT2 level was 69.0 (48.9, $109.0) \mathrm{pg} / \mathrm{mL}$. According to the $75^{\text {th }}$ percentile value of plasma SIRT2 level, we divided all the patients into highlevel group (plasma SIRT2 level $\geq 109.0 \mathrm{pg} / \mathrm{mL}$ ) and lowlevel group (plasma SIRT2 level $<109.0 \mathrm{pg} / \mathrm{mL}$ ), and clinical parameters between the two groups were compared (Table 1). Compared with the low-level group, the high-level group had significantly higher levels of C-reactive protein (CRP), blood urea nitrogen (BUN), and serum creatinine.

\section{Association between plasma SIRT2 level with indicators of AMI severity}

The indicators of AMI severity included MACE, mortality, heart failure, utilization of IABP and breathing machine, malignant arrhythmia, cardiac arrest, Killip class, left ventricular ejection fraction (LVEF), plasma brain natriuretic peptide (BNP) level, treatment, occlusive lesions, collateral circulation, as well as SYNTAX scores and GRACE scores. Compared with the low-level group (plasma SIRT2 level $<109.0 \mathrm{pg} / \mathrm{mL}$ ), the high-level group (plasma SIRT2 level $\geq 109.0 \mathrm{pg} / \mathrm{mL}$ ) was found to have higher percentage of MACE $(\mathrm{P}<0.001)$, heart failure $(\mathrm{P}<0.001)$, breathing machine use $(\mathrm{P}=0.003)$, Killip class $\geq 3(\mathrm{P}<0.001)$, LVEF $<50 \%(\mathrm{P}=0.007)$ or even $<40 \%(\mathrm{P}=0.012)$, and higher BNP level $(\mathrm{P}=0.006)$ (Table 2).

\section{Relationships between plasma SIRT2 level and clinical parameters}

Plasma SIRT2 level was noted to be associated with leukocyte and neutrophil ( $r=0.209, \mathrm{P}=0.018$ for leukocyte; $\mathrm{r}=0.217, \mathrm{P}=0.014$ for neutrophil), and also correlated with erythrocyte sedimentation rate (ESR) and CRP $(\mathrm{r}=0.215$, $\mathrm{P}=0.025$ for ESR; $\mathrm{r}=0.265, \mathrm{P}=0.004$ for CRP). Additionally, plasma SIRT2 level was also correlated with renal function $(\mathrm{r}=0.183, \mathrm{P}=0.039$ for serum creatinine; $\mathrm{r}=0.279, \mathrm{P}=0.001$ for renal dysfunction) and heart rate $(\mathrm{r}=0.199, \mathrm{P}=0.024)$. However, plasma SIRT2 level was not associated with patient's age, gender, the levels of blood glucose and lipid, cardiac Troponin-I, creatine kinase MB (CKMB), as well as GRACE and SYNTAX scores (Table 3).

\section{Univariate and multivariate Cox analysis of predictors of $M A C E$ and heart failure}

A total of 22 AMI patients had MACE recorded (including 20 cases of readmission and 16 cases of heart failure), that 2 patients died of malignant arrhythmia during hospitalization, 4 patients died of heart failure during readmission, 12 patients recovered from heart failure during readmission, and 4 patients underwent revascularization during readmission. Univariate Cox regression analysis showed that elder, renal dysfunction, higher plasma SIRT2 level, greater SYNTAX and GRACE scores, malignant arrhythmia, cardiac arrest, Killip class $\geq 3, \mathrm{LVEF}<50 \%$ or $<40 \%, \mathrm{BNP}>500 \mathrm{ng} / \mathrm{L}$, in addition to application of IABP and breathing machine were associated with higher risks of MACE and heart failure. The GRACE scores were calculated using patient's age, heart rate, systolic blood pressure, creatinine, Killip class, ST-segment deviation, elevated cardiac enzyme level, and cardiac arrest at the time of admission; for this purpose, we included GRACE and SYNTAX scores, gender, body mass index (BMI), diabetes, plasma SIRT2 level, BNP $>500 \mathrm{ng} / \mathrm{L}$, as well as utilization of IABP and breathing machine in the multivariate Cox regression analysis. Higher plasma SIRT2 level and GRACE score were associated with higher risk of MACE [for plasma SIRT2 level: hazard ratio (HR) 11.20, 95\% confidence interval (CI): 3.18-39.52, $\mathrm{P}<0.001$; for GRACE score: HR 1.03, 95\% CI: $1.02-1.05, \mathrm{P}<0.001]$ and heart failure (for plasma SIRT2 level: HR 27.10, 95\% CI: 4.65157.83, $\mathrm{P}<0.001$; for GRACE score: HR 1.03, $95 \%$ CI: 1.01-1.05, $\mathrm{P}=0.009$ ), while use of breathing machine was 
Table 1 Baseline characteristics of the AMI patients with higher and lower levels of plasma SIRT2

\begin{tabular}{|c|c|c|c|}
\hline Characteristics & SIRT2 <109.0 pg/mL (n=96) & SIRT2 $\geq 109.0 \mathrm{pg} / \mathrm{mL}(\mathrm{n}=33)$ & $P$ value \\
\hline Male, n (\%) & $73(76.0)$ & $23(69.7)$ & 0.471 \\
\hline STEMI, n (\%) & $57(59.4)$ & $17(51.5)$ & 0.431 \\
\hline Hypertension, n (\%) & $58(60.4)$ & $15(45.5)$ & 0.135 \\
\hline Previous MI, n (\%) & $11(11.5)$ & $4(12.1)$ & 0.918 \\
\hline Previous PCI, n (\%) & $12(12.5)$ & $3(9.1)$ & 0.832 \\
\hline Current smoker, n (\%) & $55(57.3)$ & $20(60.6)$ & 0.739 \\
\hline Current drinker, n (\%) & $33(34.4)$ & $7(21.2)$ & 0.158 \\
\hline Diastolic blood pressure, $\mathrm{mmHg}$ & $74.2 \pm 13.0$ & $74.2 \pm 13.3$ & 0.996 \\
\hline Body mass index, $\mathrm{kg} / \mathrm{m}^{2}$ & $25.3 \pm 2.3$ & $25.6 \pm 3.1$ & 0.655 \\
\hline C-reactive protein, $\mathrm{mg} / \mathrm{L}$ & $7.45(2.27-13.82)$ & $13.21(5.26-14.90)$ & 0.033 \\
\hline $\mathrm{ESR}, \mathrm{mm} / \mathrm{h}$ & $11.0(5.0-19.3)$ & $14.0(4.8-28.3)$ & 0.523 \\
\hline Leukocyte, $\times 10^{9} / \mathrm{L}$ & $8.93(6.92-11.07)$ & $10.21(7.88-12.15)$ & 0.056 \\
\hline Neutrophil, $\times 10^{9} / \mathrm{L}$ & $6.33(4.73-8.61)$ & $7.19(5.96-9.84)$ & 0.057 \\
\hline Lymphocyte, $\times 10^{9} / \mathrm{L}$ & $1.55(1.27-2.14)$ & $1.54(1.27-2.14)$ & 0.754 \\
\hline Hemoglobin, g/L & $130.9 \pm 15.3$ & $128.2 \pm 24.0$ & 0.543 \\
\hline LDL-C, mmol/L & $2.80(2.30-3.70)$ & $2.40(2.10-3.50)$ & 0.258 \\
\hline Triglycerides, $\mathrm{mmol} / \mathrm{L}$ & $1.29(1.00-1.80)$ & $1.43(0.95-2.33)$ & 0.764 \\
\hline Fast glucose, $\mathrm{mmol} / \mathrm{L}$ & $6.38(5.19-8.18)$ & $6.78(5.16-9.47)$ & 0.465 \\
\hline $\mathrm{HbA1C}, \%$ & $6.10(5.80-7.10)$ & $6.20(5.63-8.05)$ & 0.719 \\
\hline $\mathrm{BUN}, \mathrm{mmol} / \mathrm{L}$ & $5.65(4.62-6.87)$ & $6.67(4.60-11.62)$ & 0.038 \\
\hline Serum creatinine, $\mu \mathrm{mol} / \mathrm{L}$ & $75.1(65.0-96.4)$ & $90.0(71.6-132.1)$ & 0.012 \\
\hline $\mathrm{Na}^{+}, \mathrm{mmol} / \mathrm{L}$ & $138.6 \pm 2.5$ & $138.0 \pm 3.7$ & 0.399 \\
\hline $\mathrm{K}^{+}, \mathrm{mmol} / \mathrm{L}$ & $4.07 \pm 0.39$ & $4.23 \pm 0.42$ & 0.062 \\
\hline Homocysteine, $\mu \mathrm{mol} / \mathrm{L}$ & $17.0(12.0-21.0)$ & $16.0(12.8-28.0)$ & 0.420 \\
\hline Uric acid, $\mu \mathrm{mol} / \mathrm{L}$ & $365.5(290.3-428.8)$ & $401.5(321.5-507.5)$ & 0.064 \\
\hline Serum albumin, $g / L$ & $39.3 \pm 3.6$ & $38.0 \pm 4.2$ & 0.099 \\
\hline
\end{tabular}

Table 1 (continued) 
Table 1 (continued)

\begin{tabular}{|c|c|c|c|}
\hline Characteristics & SIRT2 <109.0 pg/mL (n=96) & SIRT2 $\geq 109.0 \mathrm{pg} / \mathrm{mL}(\mathrm{n}=33)$ & $P$ value \\
\hline Free tetraiodothyronine, $\mathrm{ng} / \mathrm{dL}$ & $1.11(1.00-1.23)$ & $1.11(1.04-1.31)$ & 0.569 \\
\hline sTSH, $\mu \mathrm{IU} / \mathrm{mL}$ & $1.13(0.74-1.91)$ & $1.28(0.63-2.18)$ & 0.809 \\
\hline Troponin-I, ng/mL & $14.6(4.3-49.5)$ & $17.5(6.8-109.2)$ & 0.337 \\
\hline Fibrinogen, mg/dL & $293.0(242.7-360.2)$ & $313.4(244.8-398.9)$ & 0.397 \\
\hline
\end{tabular}

SIRT2, sirtuin2; STEMI, ST-segment elevation myocardial infarction; MI, myocardial infarction; PCl, percutaneous coronary intervention; ESR, erythrocyte sedimentation rate; AST, aspartate aminotransferase; ALT, alanine aminotransferase; HDL-C, high density lipoprotein cholesterol; LDL-C, low density lipoprotein cholesterol; HbA1C, glycosylated Haemoglobin; BUN, blood urea nitrogen; sTSH, thyroid stimulating hormone; CKMB, creatine kinase MB.

Table 2 Comparation of AMI severity in patients with higher and lower levels of plasma SIRT2

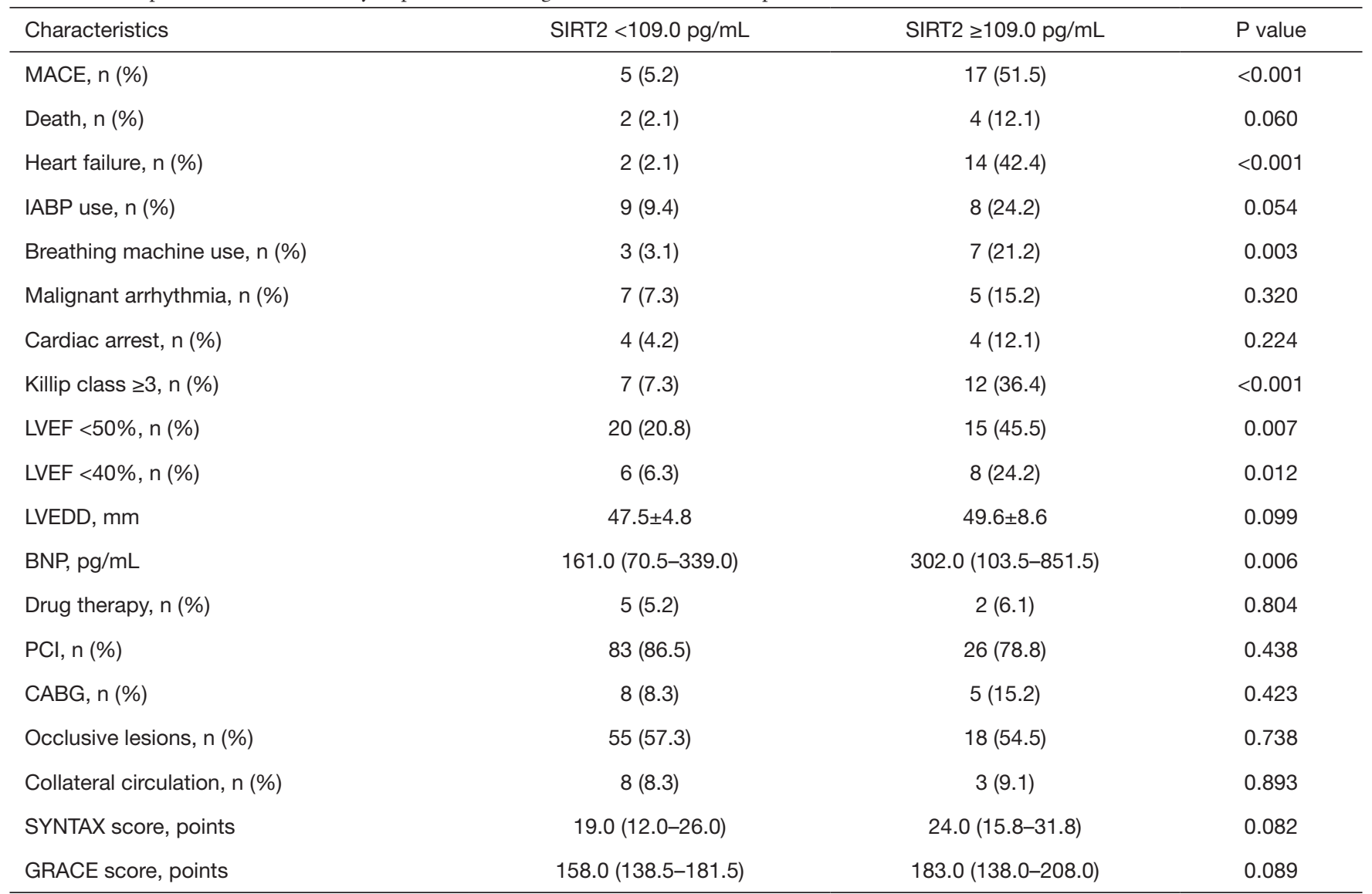

SIRT2, sirtuin2; MACE, major adverse cardiovascular event; IABP, intra-aortic balloon pump; LVEF, left ventricular ejection fraction; LVEDD, left ventricular end-diastolic diameter; BNP, brain natriuretic peptide; PCl, percutaneous coronary intervention; CABG, coronary artery bypass grafting. 
Table 3 Association between plasma SIRT2 and clinical parameters in AMI

\begin{tabular}{|c|c|c|}
\hline Characteristics & r & $P$ \\
\hline Gender $(1=$ male, $2=$ female $)$ & 0.070 & 0.430 \\
\hline Age & -0.053 & 0.550 \\
\hline Body mass index & -0.037 & 0.732 \\
\hline ESR & 0.215 & 0.025 \\
\hline C-reactive protein & 0.265 & 0.004 \\
\hline Leukocyte & 0.209 & 0.018 \\
\hline Neutrophil & 0.217 & 0.014 \\
\hline Lymphocyte & -0.061 & 0.494 \\
\hline Hemoglobin & 0.013 & 0.883 \\
\hline Platelets & 0.039 & 0.658 \\
\hline Fast glucose & 0.026 & 0.772 \\
\hline $\mathrm{HbA1C}$ & 0.026 & 0.774 \\
\hline Total cholesterol & -0.051 & 0.570 \\
\hline HDL-C & -0.124 & 0.162 \\
\hline LDL-C & -0.019 & 0.830 \\
\hline Triglycerides & 0.02 & 0.825 \\
\hline Homocysteine & 0.126 & 0.181 \\
\hline Uric acid & 0.101 & 0.255 \\
\hline Heart rate & 0.199 & 0.024 \\
\hline Systolic blood pressure & -0.039 & 0.658 \\
\hline Diastolic blood pressure & 0.065 & 0.462 \\
\hline BUN & 0.126 & 0.156 \\
\hline Serum creatinine & 0.183 & 0.039 \\
\hline Renal dysfunction & 0.279 & 0.001 \\
\hline Serum albumin & -0.069 & 0.441 \\
\hline Troponin-I & 0.127 & 0.152 \\
\hline CKMB & 0.100 & 0.278 \\
\hline GRACE score & 0.115 & 0.194 \\
\hline SYNTAX score & 0.060 & 0.496 \\
\hline Current smoking & -0.008 & 0.924 \\
\hline Current drinking & 0.014 & 0.871 \\
\hline
\end{tabular}

SIRT2, sirtuin2; AMI, acute myocardial infarction; ESR, erythrocyte sedimentation rate; HDL-C, high density lipoprotein cholesterol; LDL-C, low density lipoprotein cholesterol; BUN, blood urea nitrogen; CKMB, creatine kinase MB. associated with higher risk of MACE (HR 12.16, 95\% CI: 2.37-62.26, $\mathrm{P}=0.003$ ) and heart failure (HR 11.45, 95\% CI: 1.80-72.97, $\mathrm{P}=0.010$ ) (Table 4, Figure 1).

\section{Discussion}

In the present study, we, for the first time, assessed the role of plasma SIRT2 level in AMI patients. We found that plasma SIRT2 was an appropriate biomarker to predict heart failure and MACE after AMI. The results showed that, compared with AMI patients with lower plasma SIRT2 level, those cases with higher plasma SIRT2 level had worse cardiac function and higher risk of MACE during hospitalization and in the follow-up after discharge.

Accumulating evidence has uncovered SIRT2 played important roles in human diseases. SIRT2 showed increased levels in plasma in patients with cervical cancer compared with controls, and was considered as a potential biomarker to diagnose cervical cancer (11). SIRT2 was also increased in the peripheral blood from Alzheimer's disease (AD) subjects and elderly controls compared to levels in healthy young control, and might possibly be considered peripheral markers of AD (23). Circulating SIRT2 and other inflammatory biomarkers were significantly higher in rheumatoid arthritis (RA) patients with periodontal disease (PD) than RA without PD, indicating a augmented systemic inflammation status (24).

Previous studies demonstrated that SIRT2 was a protective factor in cardiovascular disease and also showed that expression levels of SIRT2 protein were downregulated in cardiomyocytes treated with phenylephrine or isoproterenol (7), as well as in hypertrophic hearts of mice (6) or even in hearts of T1DM rats (25). Sirt2-KO markedly exaggerated cardiac hypertrophy and fibrosis, as well as causing decreases of cardiac ejection fraction and fractional shortening in aged mice and Ang II-infused mice (6); besides, overexpression of SIRT2 attenuated agonist-induced cardiac hypertrophy in cardiomyocytes (7). Moreover, SIRT2 mediated hypertension-induced vascular remodeling (26). In cardiosurgical patients undergoing remote ischemic preconditioning, SIRT2 gene was downregulated in the cardiac tissue (8). Functional genetic variants within the SIRT2 gene promoter were found in AMI patients (9). It was previously reported that SIRT2 played a substantial role in cardiovascular disease. In addition, SIRT2 has been detected in the circulation (11), 


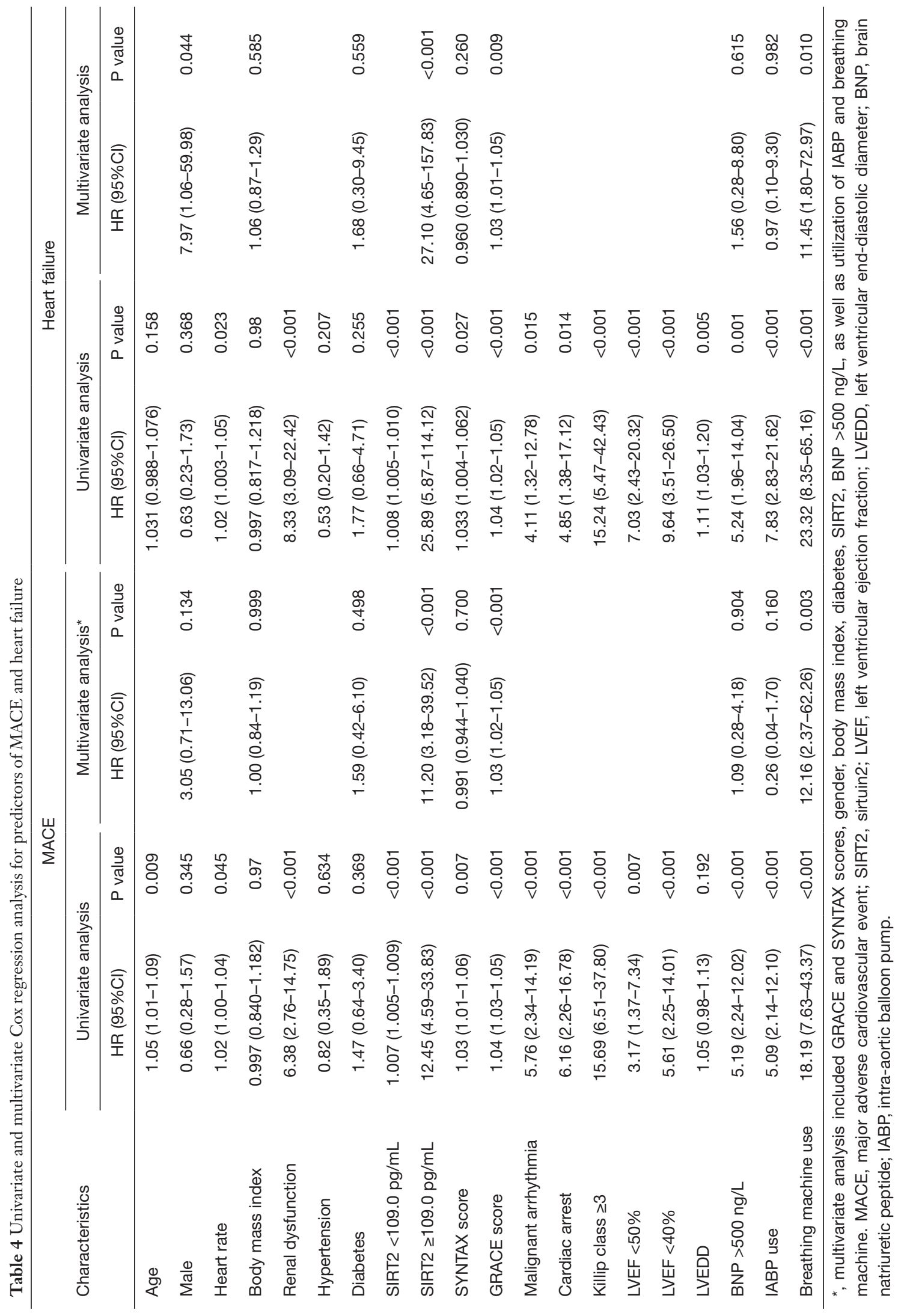



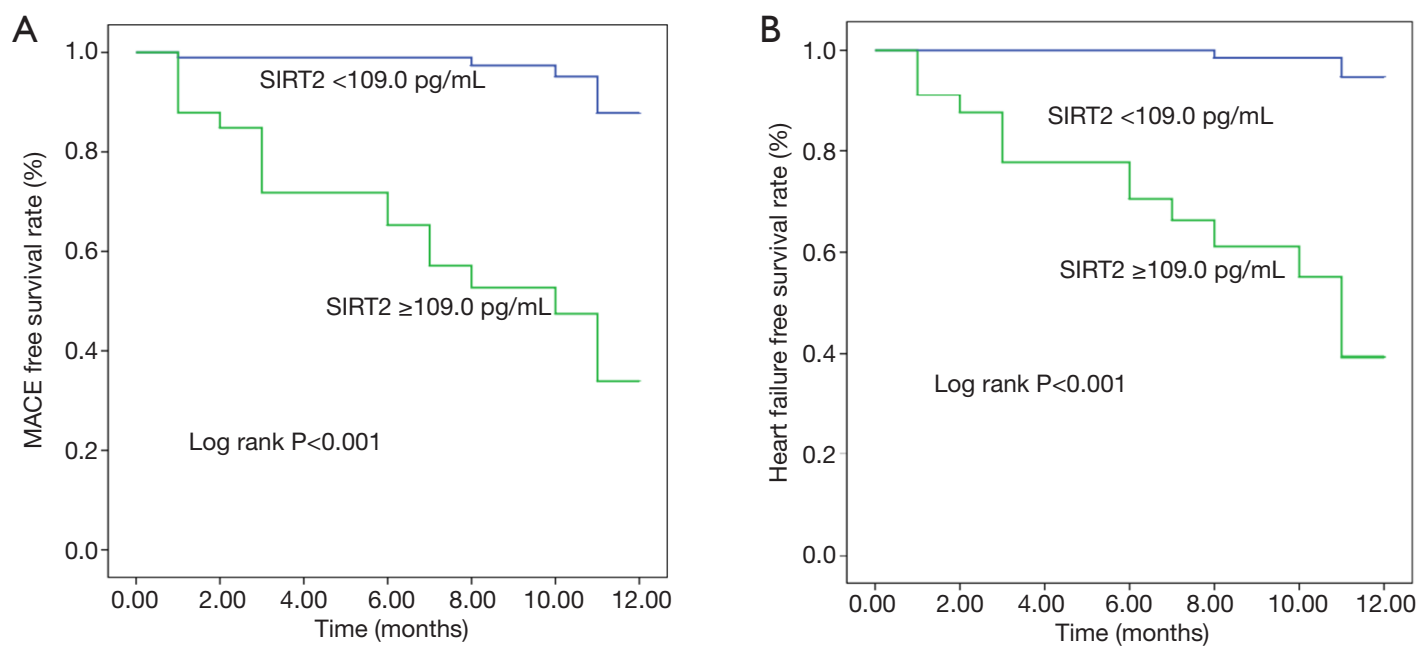

Figure 1 Kaplan-Meier curves in patients with AMI with individual levels of SIRT2 during follow-up. MACE, major adverse cardiovascular events; AMI, acute myocardial infarction; SIRT2, Sirtuin2.

however, there is no evidence about the role of circulating SIRT2 in AMI. In the present study, we noted that circulating SIRT2 was an acceptable biomarker for AMI, and the higher plasma SIRT2 level was associated with poorer AMI prognosis, especially for worse cardiac function.

In the present study, plasma SIRT2 level was found to be correlated with counts of leukocytes and neutrophils, as well as plasma levels of ESR and CRP, which were consistent with the results of previously conducted studies. Moreover, the results of present research unveiled that plasma SIRT2 level was mainly correlated with the indicators of heart failure after AMI, while that wasn't correlated with myocardial enzyme or severity of coronary artery stenosis (evaluated by SYNTAX score). We also found that in AMI patients, higher plasma SIRT2 level was associated with worse cardiac function. Inflammatory response is a key risk factor for heart failure after AMI (27). Overexpression of SIRT2 suppressed inflammatory responses and reactive oxygen species-induced macrophage cytotoxicity (2830). Inflammatory responses in AMI patients were also associated with malignant arrhythmia $(31,32)$, and higher plasma SIRT2 level was noted to have higher percentage of malignant arrhythmia and cardiac arrest in the present study.

Additionally, SIRT2 was reported to be associated with renal inflammatory injury, in which SIRT2 showed an antiinflammatory effect through regulating p65 binding to the promoters of CXCL2 and CCL2 (14). In the current study, plasma SIRT2 level was correlated with renal function in AMI, which might be due to the inflammatory responses in AMI patients.

The present study contains a number of limitations: (I) the period of follow-up was short, therefore, long-term follow-up studies need to be conducted to further analyze the association between plasma SIRT2 level and AMI; (II) this is a study with small sample size; large sample size should be carried out to verify our findings.

\section{Conclusions}

Our findings revealed that plasma SIRT2 level was a proper biomarker to predict heart failure and MACE after AMI, which, as indicated by previous studies, might be through regulating metabolic and inflammatory pathways.

\section{Acknowledgments}

Funding: This research was supported by Natural Science Foundation of China (No. 81800304).

\section{Footnote}

Reporting Checklist: The authors have completed the STROBE reporting checklist. Available at http://dx.doi. org/10.21037/jtd-20-2234

Data Sharing Statement: Available at http://dx.doi. 
org/10.21037/jtd-20-2234

Peer Review File: Available at http://dx.doi.org/10.21037/jtd20-2234

Conflicts of Interest: All authors have completed the ICMJE uniform disclosure form (available at http://dx.doi. org/10.21037/jtd-20-2234). The authors have no conflicts of interest to declare.

Ethical Statement: The authors are accountable for all aspects of the work in ensuring that questions related to the accuracy or integrity of any part of the work are appropriately investigated and resolved. The study was conducted in accordance with the Declaration of Helsinki (as revised in 2013). The study was approved by the Ethics Committee of Beijing Chao-Yang Hospital (No. 2018-2-7-3) and informed consent was taken from all the patients.

Open Access Statement: This is an Open Access article distributed in accordance with the Creative Commons Attribution-NonCommercial-NoDerivs 4.0 International License (CC BY-NC-ND 4.0), which permits the noncommercial replication and distribution of the article with the strict proviso that no changes or edits are made and the original work is properly cited (including links to both the formal publication through the relevant DOI and the license). See: https://creativecommons.org/licenses/by-nc-nd/4.0/.

\section{References}

1. Bonkowski MS, Sinclair DA. Slowing ageing by design: the rise of $\mathrm{NAD}(+)$ and sirtuin-activating compounds. Nat Rev Mol Cell Biol 2016;17:679-90.

2. Winnik S, Auwerx J, Sinclair DA, et al. Protective effects of sirtuins in cardiovascular diseases: from bench to bedside. Eur Heart J 2015;36:3404-12.

3. Yamamoto H, Schoonjans K, Auwerx J. Sirtuin functions in health and disease. Mol Endocrinol 2007;21:1745-55.

4. Donmez G, Outeiro TF. SIRT1 and SIRT2: emerging targets in neurodegeneration. EMBO Mol Med 2013;5:344-52.

5. Matsushima S, Sadoshima J. The role of sirtuins in cardiac disease. Am J Physiol Heart Circ Physiol 2015;309:H1375-89.

6. Tang X, Chen XF, Wang NY, et al. SIRT2 Acts as a Cardioprotective Deacetylase in Pathological Cardiac Hypertrophy. Circulation 2017;136:2051-67.
7. Sarikhani M, Maity S, Mishra S, et al. SIRT2 deacetylase represses NFAT transcription factor to maintain cardiac homeostasis. J Biol Chem 2018;293:5281-94.

8. Zitta K, Meybohm P, Gruenewald M, et al. Profiling of cell stress protein expression in cardiac tissue of cardiosurgical patients undergoing remote ischemic preconditioning: implications for thioredoxin in cardioprotection. J Transl Med 2015;13:34.

9. Yang W, Gao F, Zhang P, et al. Functional genetic variants within the SIRT2 gene promoter in acute myocardial infarction. PLoS One 2017;12:e0176245.

10. Gomes P, Fleming Outeiro T, Cavadas C. Emerging Role of Sirtuin 2 in the Regulation of Mammalian Metabolism. Trends Pharmacol Sci 2015;36:756-68.

11. Berggrund M, Enroth S, Lundberg M, et al. Identification of Candidate Plasma Protein Biomarkers for Cervical Cancer Using the Multiplex Proximity Extension Assay. Mol Cell Proteomics 2019;18:735-43.

12. Yuan F, Xu ZM, Lu LY, et al. SIRT2 inhibition exacerbates neuroinflammation and blood-brain barrier disruption in experimental traumatic brain injury by enhancing NFkappaB p65 acetylation and activation. J Neurochem 2016;136:581-93.

13. Rothgiesser KM, Erener S, Waibel S, et al. SIRT2 regulates NF-kappaB dependent gene expression through deacetylation of p65 Lys310. J Cell Sci 2010;123:4251-8.

14. Jung YJ, Lee AS, Nguyen-Thanh T, et al. SIRT2 Regulates LPS-Induced Renal Tubular CXCL2 and CCL2 Expression. J Am Soc Nephrol 2015;26:1549-60.

15. Wang YP, Zhou LS, Zhao YZ, et al. Regulation of G6PD acetylation by SIRT2 and KAT9 modulates NADPH homeostasis and cell survival during oxidative stress. EMBO J 2014;33:1304-20.

16. German NJ, Haigis MC. Sirtuins and the Metabolic Hurdles in Cancer. Curr Biol 2015;25:R569-83.

17. Weil BR, Neelamegham S. Selectins and Immune Cells in Acute Myocardial Infarction and Post-infarction Ventricular Remodeling: Pathophysiology and Novel Treatments. Front Immunol 2019;10:300.

18. Lim GB. Acute coronary syndromes: Supplemental oxygen in myocardial infarction. Nat Rev Cardiol 2017;14:632.

19. Synetos A, Papanikolaou A, Toutouzas K, et al. Metabolic syndrome predicts plaque rupture in patients with acute myocardial infarction. An optical coherence study. Int J Cardiol 2016;209:139-41.

20. Ye F, Winchester D, Jansen M, et al. Assessing Prognosis of Acute Coronary Syndrome in Recent Clinical Trials: A Systematic Review. Clin Med Res 2019;17:11-9. 
21. Davis WT, Montrief T, Koyfman A, et al. Dysrhythmias and heart failure complicating acute myocardial infarction: An emergency medicine review. Am J Emerg Med 2019;37:1554-61.

22. World Medical A. World Medical Association Declaration of Helsinki: ethical principles for medical research involving human subjects. JAMA 2013;310:2191-4.

23. Wongchitrat P, Pakpian N, Kitidee K, et al. Alterations in the Expression of Amyloid Precursor Protein Cleaving Enzymes mRNA in Alzheimer Peripheral Blood. Curr Alzheimer Res 2019;16:29-38.

24. Panezai J, Ali A, Ghaffar A, et al. Upregulation of circulating inflammatory biomarkers under the influence of periodontal disease in rheumatoid arthritis patients. Cytokine 2020;131:155117.

25. Yuan Q, Zhan L, Zhou QY, et al. SIRT2 regulates microtubule stabilization in diabetic cardiomyopathy. Eur J Pharmacol 2015;764:554-61.

26. Hashimoto-Komatsu A, Hirase T, Asaka M, et al. Angiotensin II induces microtubule reorganization mediated by a deacetylase SIRT2 in endothelial cells.

Cite this article as: Zheng M, Du X, Zhao L, Sun H, Chen M, Yang X. Elevated plasma Sirtuin2 level predicts heart failure after acute myocardial infarction. J Thorac Dis 2021;13(1):5059. doi: $10.21037 /$ jtd-20-2234
Hypertens Res 2011;34:949-56.

27. Nahrendorf M, Frantz S, Swirski FK, et al. Imaging systemic inflammatory networks in ischemic heart disease. J Am Coll Cardiol 2015;65:1583-91.

28. Kim MJ, Kim DW, Park JH, et al. PEP-1-SIRT2 inhibits inflammatory response and oxidative stress-induced cell death via expression of antioxidant enzymes in murine macrophages. Free Radic Biol Med 2013;63:432-45.

29. Eskandarian HA, Impens F, Nahori MA, et al. A role for SIRT2-dependent histone H3K18 deacetylation in bacterial infection. Science 2013;341:1238858.

30. Pais TF, Szego EM, Marques O, et al. The NADdependent deacetylase sirtuin 2 is a suppressor of microglial activation and brain inflammation. EMBO J 2013;32:2603-16.

31. Kobayashi Y. How to manage various arrhythmias and sudden cardiac death in the cardiovascular intensive care. J Intensive Care 2018;6:23.

32. Frangogiannis NG. The inflammatory response in myocardial injury, repair, and remodelling. Nat Rev Cardiol 2014;11:255-65. 